\title{
Evaluation of the Performance of Health Extension Workers in HIV-1/2 Screening Tests: A Comparative Cross-Sectional Study, Addis Ababa, Ethiopia
}

\author{
Mulatu Gashaw $\mathbb{D}^{\mathrm{D}},{ }^{1}$ Samuel Kindie, ${ }^{2}$ Minale Fekadie $\mathbb{D}^{\mathbb{D}},{ }^{3}$ Kassu Desta, ${ }^{2}$ and Dawit Wolday ${ }^{4,5}$ \\ ${ }^{1}$ School of Medical Laboratory Science, Jimma University, Jimma, Ethiopia \\ ${ }^{2}$ Department of Medical Laboratory Science, Addis Ababa University, Addis Ababa, Ethiopia \\ ${ }^{3}$ School of Biomedical Sciences, Jimma University, Jimma, Ethiopia \\ ${ }^{4}$ Faculty of Medicine, Mekele University, Mek'ele, Ethiopia \\ ${ }^{5}$ Medical Biotech Laboratories, Addis Ababa, Ethiopia \\ Correspondence should be addressed to Mulatu Gashaw; mulatugashaw@gmail.com
}

Received 9 June 2020; Revised 22 October 2020; Accepted 10 November 2020; Published 20 November 2020

Academic Editor: Glenda Gray

Copyright (c) 2020 Mulatu Gashaw et al. This is an open access article distributed under the Creative Commons Attribution License, which permits unrestricted use, distribution, and reproduction in any medium, provided the original work is properly cited.

\begin{abstract}
Background. Human resources for health-care delivery are essential for attaining global health and development goals. Especially in developing countries, health extension workers are frontline health personnel who can play a key role in preventing and controlling HIV/AIDS. This study aimed to evaluate the performance of health extension workers in HIV-1/2 screening tests. Methodology. A comparative cross-sectional study was carried out to evaluate the performance of health extension workers in HIV-1/2 screening tests. Study participants had performed HIV screening tests on the prepared sample panels. Finally, the percentage of accuracy, error rate, sensitivity, specificity, predictive values, and measure of agreement (kappa) were calculated using SPSS version 26. Result. Totally, 1600 HIV screening tests were performed, and of these, 684 and 235 tests were done by HEWs $(n=15)$ and laboratory personnel $(n=5)$, respectively, with three discordant results by HEWs from a single sample panel which was weak reactive for HIV antibody test. The sensitivity, specificity, PPV, and NPV of HIV screening tests by HEWs were $97.4 \%, 100 \%, 100 \%$, and $97.22 \%$, respectively, and 100\% for all parameters when it is tested by laboratory professionals. The measure of kappa agreement was 0.971 (95\% CI, 0.932-1) for HEWs and 1 for laboratory personnel compared with the reference result. Conclusion. Based on this evidence, we conclude that the potential contribution of HEWs can be invaluable in the expansion of HIV screening tests nationwide to compensate the shortage of laboratory personnel.
\end{abstract}

\section{Background}

Human resources for health-care delivery are essential for attaining global health and development goals $[1,2]$. There is a strong correlation between the density of the health workforce, service coverage, and positive health outcomes, and a shortage of health workers has been widely and consistently identified as a barrier to the delivery of health services [1-4]. The HIV pandemic has increased the demand of health workers, and at the same time, the increased global commitment to HIV puts more pressure on bottlenecks created by health workforce shortages [5].
Health extension is an approach of promoting change through demonstration, working with opinion leaders and community-based educational activities [6]. Health extension workers (HEWs) are primarily responsible to achieve this program by working with the families and community at a grass root level to promote health and prevent disease through awareness creation [7]. Therefore, HEWs are frontline health personnel who can play a key role in increasing HIV/AIDS diagnostic services in the community $[8,9]$.

Rapid HIV screening tests have been developed predominantly for quick, easy to use, reliable onsite antibody 
testing for HIV by nonlaboratory-trained health professionals. The introduction of HIV screening tests to resourcelimited countries could resolve many logistical issues [10]. Currently, HIV screening tests are widely used in nonlaboratory settings by nonlaboratory-trained operators [11]. Human immunodeficiency virus (HIV) screening testing is a key tool to fight against HIV/AIDS pandemic in low-income countries. However, a quality assurance program is very essential to ensure the quality of HIV screening test service outcomes [12-14]. Different research studies showed that transport costs are often a barrier to access VCT. Therefore, HIV prevention and control programs that aim in helping more people should consider home-based VCT services, especially in resource-limited settings where transport to a clinic and confidentiality are considerable barriers to access the service [15-17].

In countries, severely affected by HIV/AIDS pandemic, shortage of health workers present a major obstacle to scaling up HIV services [1]. To cope with the existing needs, nonlaboratory personnel should be participated in performing HIV screening tests [18]. Therefore, this study aimed to evaluate the performance of HIV screening tests by health extension workers in comparison with laboratory personnel.

\section{Methods}

2.1. Study Design and Setting. A comparative cross-sectional study design was carried out to evaluate the performance of HIV-1/2 screening tests by health extension workers compared with laboratory personnel in Addis Ababa, Ethiopia.

2.2. Study Participants. From 1375 health extension workers in the city, 15 HEWs from two subcities and five laboratory personnel from Addis Ababa University were taken conveniently. A total of twenty participants (HEWs $=15$ and laboratory personnel $=5$ ) were enrolled in study, and every one of them were performed HIV screening tests on 40 test panels based on the national HIV test algorithm of Ethiopia, and a total of 919 tests were done by both groups.

2.3. Panel Preparation and Dispatching. Ninety-two leftover sera samples were collected from St. Paul Hospital found in Addis Ababa and tested using rapid HIV test kits. Once the result was known, the sera were pooled, and a totally of forty sample panels were prepared. All the prepared sample panels were confirmed by fourth-generation ELISA, Murex HIV Ag/Ab Combination (Bio Murex, UK), for their HIV result again before used for the study. Finally, thirty-five negative and five positive sample panels were prepared to be tested by study participants and stored at $2-8^{\circ} \mathrm{C}$ till being tested by the participants.

2.4. Data Collection. After short demonstration about rapid HIV testing was given to HEWs, they have examined all 40 panels for the presence or absence of HIV-1/2 antibodies using three rapid HIV test kits according to the national HIV test algorithm, and finally they have recorded and reported their result to the investigators.

2.5. Data Analysis. Reported data were compared with the reference result and categorized as correct or incorrect and entered in Microsoft Excel sheet and transferred to SPSS version 26 for analysis. The kappa measure of agreement test, sensitivity, specificity, positive predictive value, negative predictive value, percentages of correct test results, and error rates were analyzed using SPSS software. Based on the calculated results, error rate $<5 \%$ between each rapid test kit was considered as tolerable.

2.6. Ethical Consideration. Ethical approval was obtained from Department Research and Ethical Review Committee (DRERC) of Medical Laboratory Science, and the participants were asked to fill the written informed consent prior to enrolling in the study.

\section{Results}

3.1. Sociodemographic Characteristics of Participants. A total of 20 participants were enrolled in the study, and all of them have responded as they were very confident and comfortable to perform HIV screening tests. They also responded that their preference of training was demonstration in person. All health extension workers have taken at least one HIV-related training (Table 1), and their performance in HIV screening tests was correct except three of the HEWs. Of those HEWs, who had incorrect HIV screening tests, one has taken all the listed trainings related to $\mathrm{HIV}$, and the other two took counseling and VCT (Table 1).

\subsection{Performance of HEWs on Rapid HIV Tests against Lab-} oratory Personnel. All HIV screening tests done by health extension workers, and skilled laboratory personnel were evaluated against the reference result which was confirmed by fourth-generation ELISA, Murex HIV Ag/Ab Combination (Bio Murex, UK). The performance of laboratory personnel was $100 \%$ for all parameters, while the performance of health extension workers was calculated, and the sensitivity, specificity, positive predictive value, and negative predictive were $97.4 \%$ (95\% CI, 97-100\%), 100\%, 100\%, and $97.2 \%$, respectively, for all sample panels (Table 2). The kappa agreement of HEWs with laboratory personnel and reference result is $0.97(95 \% \mathrm{CI}, 0.9-1)$ which shows a perfect agreement (Table 2).

Totally, nine hundred and nineteen (919) rapid HIV tests were performed and reported with 916 (99.7\%) concordance and $3(0.3 \%)$ discordance results from a single sample panel by three of the health extension workers comparing with reference results using three rapid HIV test kits. The panel which was misdiagnosed by health extension workers was positive (reactive by any of the test kits) with faint red line, but both lines are not the same intensity that is normally taken as weak reactive. Except for those three health extension workers who scored a single error results each, all the 
TABLE 1: Proportions of health extension workers who have taken HIV-related training before and percentage of their performance.

\begin{tabular}{lccc}
\hline HIV-related training & No. of participants (\%) & Percentage of concordant results no. (\%) & Error rate (\%) \\
\hline Participants took all training & $4(26.7)$ & $179(99.4)$ & $45(100)$ \\
Participants took all except testing & $1(6.7)$ & $190(100)$ & 0.6 \\
Counseling, VCT and PMTCT & $4(26.7)$ & $133(98.5)$ & 0 \\
Counseling and VCT & $3(20.0)$ & $47(100)$ & 1.5 \\
Counseling & $1(6.7)$ & $90(100)$ & 0 \\
VCT & $2(13.3)$ & $684(99.6)$ & 0 \\
Total & $15(100)$ & 0.4 \\
\hline
\end{tabular}

TABLE 2: Comparing group performance of health extension workers with laboratory professionals on HIV rapid tests *.

\begin{tabular}{lccccc}
\hline Groups & Sensitivity (\%) & Specificity (\%) & PPV (\%) & NPV (\%) & Kappa agreement \\
\hline HEWs & $97.4(97-100)$ & 100 & 100 & 97.2 & $0.97(0.9-1)$ \\
Lab. professionals & 100 & 100 & 100 & 100 & 1 \\
\hline
\end{tabular}

* Against gold standard as assessed using ELISA, $a$. All laboratory personnel and twelve HEWs who did not have error results, $b$. Three HEWs who have made one error result in each.

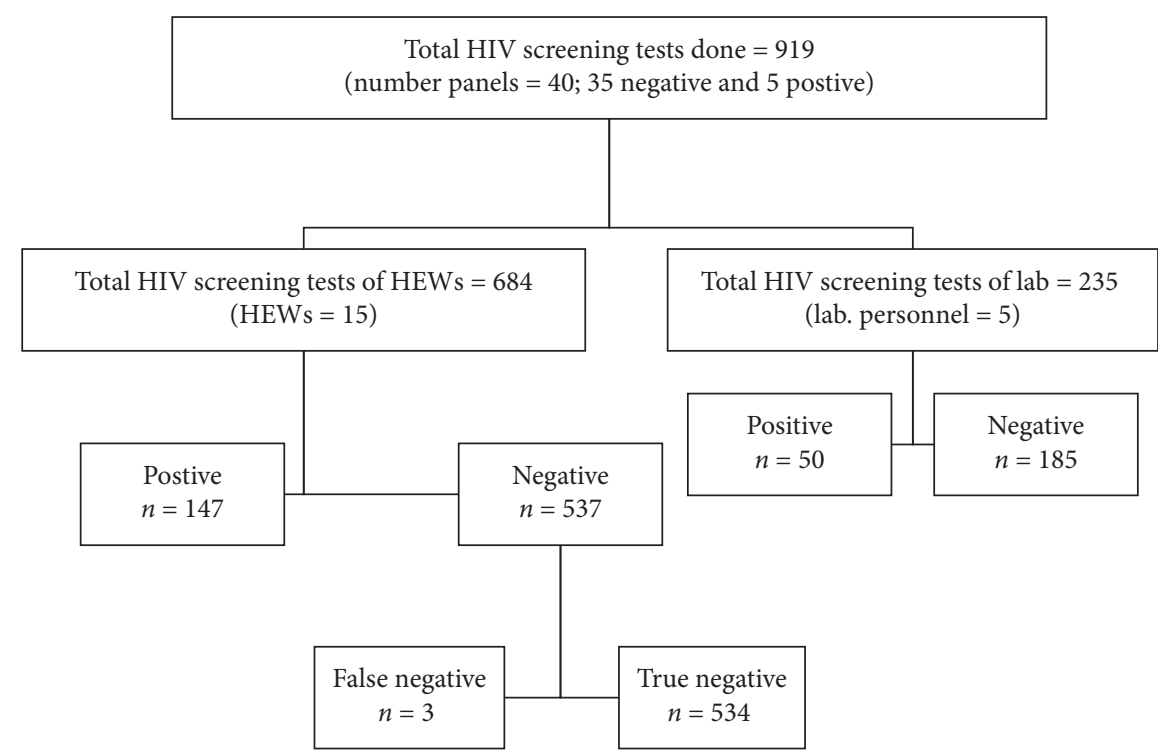

FIgURE 1: Prototypical STARD diagram to report HIV screening test results of health extension workers and laboratory personnel on pooled panels.

others $(n=12)$ scored concordant results with laboratory personnel and reference results (Figure 1).

\section{Discussion}

According to $\mathrm{WHO}$, the minimum required specificity and sensitivity for rapid HIV screening tests are at least 98\%, and $99 \%$, respectively $[13,19]$. The current study showed that the overall performance of health extension workers on these rapid HIV screening tests is comparable with WHO recommendations. On the other hand, the sensitivity and specificity of HEWs on rapid HIV screening tests were greater than the study done in other African countries (sensitivity $=92.5 \%$ and specificity $=97.5 \%$ ) [20]. These differences in HIV screening test performance between health extension workers and laboratory personnel were probably due to lack of experience for weakly reactive samples.

Even though different research studies showed that rapid HIV screening tests could be performed and read with accuracy by nonlaboratory personnel [12, 21, 22], our finding showed that all three errors were reported by HEWs from a single sample panel with faint red line which is commonly called as weak reactive. This finding is agreed with a study done in Congo which showed that, with a proper training, nurse counselors' produced false-negative results in weakly reactive samples [23].

An other study done in South Africa showed that the sensitivity and specificity of rapid HIV screening tests performed by nurses/counselors were $92.5-97.3 \%$ and 97.6-98.2\%, respectively, but $100 \%$ for all parameters when performed by laboratory technicians [24], which was 
comparable with our finding. The discrepancy in test performance between nonlaboratory personnel and laboratory personnel was probably due to a lack of experience in weak reactive samples.

In our finding, the concordance and discordance test results of HEWs are $99.6 \%$ and $0.4 \%$, respectively which is in agreement with studies done in African countries by Plate et al. who reported that the concordance between onsite rapid HIV screening test results, and results by laboratory personnel ranged from 95.7-99.5\% (median: 98.7\%) [20]. The discordance rate of our finding $(0.4 \%)$ was less than the research studies done in other African countries and United States of America whose discordance rate was ranged from $0.4-1.1 \%$ and $2.1-4.6 \%$, respectively $[20,25]$. The error rate $(0.4 \%)$ of our finding is also less than WHO recommendations, which says that it should not exceed 5\% [13]. Therefore, the performance of HEWs in our study is acceptable to perform rapid HIV screening tests.

\section{Conclusion}

Based on the evidence from the current study, we conclude that where there is the necessary support, the potential contribution of HEWs can be optimized and represents a valuable addition to the urgent expansion of human resources for health, specifically for HIV services, nationwide.

\section{Data Availability}

The data used to support the findings of this study are included in the manuscript.

\section{Conflicts of Interest}

The authors declare no conflicts of interest.

\section{Acknowledgments}

The authors are grateful to Addis Ababa University and Health extension workers for their willingness to do this research.

\section{References}

[1] Organization WH, Global Strategy on Human Resources for Health: Workforce 2030, World Health Organization, Geneva, Switzerland, 2016.

[2] S. M. Kabene, C. Orchard, J. M. Howard, M. A. Soriano, and R. Leduc, "The importance of human resources management in health care: a global context," Human Resources for Health, vol. 4, no. 1, p. 20, 2006.

[3] M. Taegtmeyer, T. Martineau, J. H. Namwebya et al., "A qualitative exploration of the human resource policy implications of voluntary counselling and testing scale-up in Kenya: applying a model for policy analysis," BMC Public Health, vol. 11, no. 1, p. 812, 2011.

[4] B. Kebede, T. Abate, and D. Mekonnen, "HIV self-testing practices among health care workers: feasibility and options for accelerating HIV testing services in Ethiopia," Pan African Medical Journal, vol. 15, no. 1, 2013.
[5] Organization WH, Treat, Train, Retain: the AIDS and Health Workforce Plan: Report on the Consultation on AIDS and Human Resources for Health, WHO, Geneva, Switzerland, 2006.

[6] A. Medhanyie, M. Spigt, Y. Kifle et al., "The role of health extension workers in improving utilization of maternal health services in rural areas in Ethiopia: a cross sectional study," BMC Health Services Research, vol. 12, no. 1, p. 352, 2012.

[7] S. Singh, S. Upadhyaya, P. Deshmukh et al., "Time motion study using mixed methods to assess service delivery by frontline health workers from South India: methods," Human Resources for Health, vol. 16, no. 1, p. 17, 2018.

[8] M. Yazachew and Y. Alen, "Introduction to health education," in Ethiopia Public Health Training InitiativeAddis Ababa University, Addis Ababa, Ethiopia, 2004.

[9] Y. Hailu, A. Tekilegiorgis, and A. Aga, "Know-how of primary eye care among health extension workers (HEWs) in Southern Ethiopia," Ethiopian Journal of Health Development, vol. 23, no. 2, 2009.

[10] V. Puro, D. Francisci, L. Sighinolfi et al., "Benefits of a rapid HIV test for evaluation of the source patient after occupational exposure of healthcare workers," Journal of Hospital Infection, vol. 57, no. 2, pp. 179-182, 2004.

[11] Y.-H. C. Chiu, J. Ong, S. Walker et al., "Photographed rapid HIV test results pilot novel quality assessment and training schemes," PLoS One, vol. 6, no. 3, 2011.

[12] K. Yao, W. Wafula, E. C. Bile et al., "Ensuring the quality of HIV rapid testing in resource-poor countries using a systematic approach to training," American Journal of Clinical Pathology, vol. 134, no. 4, pp. 568-572, 2010.

[13] Organization WH and Control CfD, Guidelines for Using HIV Testing Technologies in Surveillance: Selection, Evaluation, and Implementation, World Health Organization, Geneva, Switzerland, 2001.

[14] M. F. Afework, K. Admassu, A. Mekonnen, S. Hagos, M. Asegid, and S. Ahmed, "Effect of an innovative community based health program on maternal health service utilization in North and South Central Ethiopia: a community based cross sectional study," Reproductive Health, vol. 11, no. 1, p. 28, 2014.

[15] P. S. Yoder, A. R. Katahoire, D. Kyaddondo, Z. Akol, R. Bunnell, and F. Kaharuza, Home-Based HIV Testing and Counselling in a Survey Context in Uganda, DHS Qualitative Research Studies NO. 12, Calverton, MD, USA, 2006.

[16] W. Were, J. Mermin, R. Bunnell, J. P. Ekwaru, and F. Kaharuza, "Home-based model for HIV voluntary counselling and testing," The Lancet, vol. 361, no. 9368, p. 1569, 2003.

[17] S. Campbell and R. Klein, "Home testing to detect human immunodeficiency virus: boon or bane?" Journal of Clinical Microbiology, vol. 44, no. 10, pp. 3473-3476, 2006.

[18] S. O. Baine, A. Kasangaki, and E. M. M. Baine, "Task shifting in health service delivery from a decision and policy makers' perspective: a case of Uganda," Human Resources for Health, vol. 16, no. 1, p. 20, 2018.

[19] Cosponsors U, Guidelines for using HIV testing technologies in surveillance: selection, evaluation and implementation. learning, 2009.

[20] D. K. Plate and Group RHTEW, "Evaluation and implementation of rapid HIV tests: the experience in 11 African countries," AIDS Research and Human Retroviruses, vol. 23, no. 12, pp. 1491-1498, 2007.

[21] K. E. Bowles, H. A. Clark, E. Tai et al., "Implementing rapid HIV testing in outreach and community settings: results from 
an advancing HIV prevention demonstration project conducted in seven U.S. Cities," Public Health Reports, vol. 123, no. 3_suppl, pp. 78-85, 2008.

[22] D. B. Everett, K. Baisley, J. Changalucha et al., "Suitability of simple human immunodeficiency virus rapid tests in clinical trials in community-based clinic settings," Journal of Clinical Microbiology, vol. 47, no. 4, pp. 1058-1062, 2009.

[23] D. B. Klarkowski, J. M. Wazome, K. M. Lokuge, L. Shanks, C. F. Mills, and D. P. O'Brien, "The evaluation of a rapid in situ HIV confirmation test in a programme with a high failure rate of the WHO HIV two-test diagnostic algorithm," PLoS One, vol. 4, no. 2, 2009.

[24] D. Moodley, P. Moodley, T. Ndabandaba, and T. Esterhuizen, "Reliability of HIV rapid tests is user dependent," South African Medical Journal = Suid-Afrikaanse Tydskrif Vir Geneeskunde, vol. 98, no. 9, pp. 707-709, 2008.

[25] T. C. Granade, B. S. Parekh, S. K. Phillips, and J. S. McDougal, "Performance of the OraQuick and Hema-strip rapid HIV antibody detection assays by non-laboratorians," Journal of Clinical Virology, vol. 30, no. 3, pp. 229-232, 2004. 